\title{
Curso Híbrido usando a Rede Social Facebook no Ensino de Programação de Computadores
}

\author{
Eulália C. da Mata ${ }^{1}$, Márcia F. Pinheiro ${ }^{1}$, Antônio F. L. Jacob Jr. ${ }^{1}$, João C. W. A. \\ Costa $^{1}$, Ádamo L. de Santana ${ }^{1}$, Carlos R. L. Francês ${ }^{1}$ \\ ${ }^{1}$ Instituto de Tecnologia - Universidade Federal do Pará (UFPA) \\ Caixa Postal 479 - 66.075-110 - Belém - PA - Brazil \\ \{eucmata, eng.marciafontes\}@gmail.com, \{jacobjr, jweyl, adamo, \\ rfrances\}@ufpa.br
}

\begin{abstract}
This article presents a pilot study of hybrid course based on the interactions and connections that occurred in a group of social network Facebook for middle school students. In this context of attracting new students to the field of computing, if realized in the network analysis applications for other individuals to participate in the online course, besides the relations between the actors and flows that create bonds of communication and information exchange.
\end{abstract}

Resumo. Este artigo apresenta uma pesquisa-piloto de curso hibrido com base nas interações e conexões que ocorreram em um grupo da rede social Facebook para alunos de ensino médio. Nesse contexto de atrair novos alunos para a área da computação, perceberam-se na análise de rede os pedidos de outros indivíduos para participarem do curso online, além das relações e fluxos entre os atores que criram laços de comunicação e troca de informações.

\section{Introdução}

A internet brasileira já teve acesso restrito, aberto somente às iniciativas acadêmicas até pelo menos 1994 via RPN (Rede Nacional de Pesquisas). Porém, havia uma exceção a essa regra, o Alternex, um serviço de troca de emails e conferências eletrônicas, voltado para a sociedade civil, que em 1989 foi adquirido pelo Ibase (Instituto Brasileiro de Análises Sociais e Econômicas) [Falavigna 2011].

Diferente do inicio da história da internet no Brasil, o acesso as Tecnologias de Informação e Comunicação (TIC) estão cada vez mais acessíveis à sociedade civil. Acompanhando este avanço o ambiente escolar passa por transformações com a inserção de tecnologias que visam atender os aspectos de interação e aprendizado entre todos os atores envolvidos nesse sistema. Segundo [Nascimento e Hetkowski 2009] a comunicação e a educação são inseparáveis, apesar de serem áreas distintas. Complementando essa idéia [Kenski 2008] ressalta que o ato de comunicação em educação consiste em um meio para construir conhecimento.

As mídias sociais são apontadas como tendência na vida de universitários e professores dentro de uma perspectiva para o ensino superior no mundo, em um prazo de até 2 anos entre 2014 e 2015 [Johnson et al 2014]. Os aspectos em destaque do uso 
de mídias sociais associadas à educação são a facilidade na comunicação, a familiaridade com a tecnologia e o apoio a trocar conteúdos estudados [Johnson et al 2014]. As perspectivas tecnológicas brasileiras para o ensino fundamental e médio até 2017 abordam a inclusão do ensino online, o aprendizado hibrido e os modelos colaborativos por meio de mudanças dos paradigmas da educação [Johnson et al 2012].

As redes sociais destacam-se pelo número de pessoas ativas nesse tipo de plataforma [Lima 2014]; pela mobilidade ao acesso; pelas interações sociais; por integrarem informações de forma multisensorial [Maness 2006], através de imagens, textos, vídeos e áudios; por fomentarem produções colaborativas; além de serem meio de articulação dos fenômenos de mobilizações e pela possibilidade de uso em processo de ensino aprendizagem.

Uma das principais dificuldades em plena era digital consiste no ensino de programação de computadores e na formação de profissionais na área da Ciência da Computação [Bennedsen e Caspersen 2009]. Há uma lacuna no sistema de ensino superior, no qual apresenta um alto índice de evasão nos primeiros anos dos cursos da área da computação. Em geral os fatores para evasão são por falta de condições financeiras para se manter estudando, desconhecimento do curso, formação básica ineficiente, mudança de residência, dificuldades em compreender os assuntos abordados nas disciplinas básicas entre outros [Lobo 2012].

Este artigo converge para o problema de desconhecimento do curso de computação entre alunos do ensino médio e na compreensão sobre programação de computadores. O objetivo dessa pesquisa consiste em apresentar o ensino hibrido que contemplou momentos presenciais (off-line) em laboratório de informática e momentos online em rede social, atendendo alunos de ensino médio, em pesquisa-piloto do projeto de "Capacitação de alunos de Ensino Médio por meio de um Sistema Lúdico e Colaborativo visando à participação em uma Olimpíada de Programação de Computadores" e analise do comportamento na rede social facebook. Para [Scaico e Queiroz 2013] as tecnologias atraem as pessoas a interagirem, se expressarem, acessarem o conhecimento e aprenderem novas coisas por meio de uma forte dinâmica social.

Este trabalho está organizado da seguinte forma: na seção 2 é apresentada uma visão geral sobre "Aprendizagem Híbrida" e conceitos relacionados. Na seção 3, trata-se da experiência no projeto de ensino de programação de computadores a alunos de ensino médio. Na seção 4 uma analise dos resultados apresentados e as contribuições. Na seção 5, as considerações finais e uma breve descrição dos trabalhos futuros.

\section{Aprendizagem Híbrida (Blended Learning)}

A aprendizagem híbrida consiste em manter as melhores características de um processo de aprendizagem realizado em sala de aula (t-learning) associada com as melhores características de um processo de aprendizagem online (e-learning) [Mohammad 2009]. Realiza-se o uso de diversas ferramentas como: wiki, blogs, redes sociais, celulares e computadores como instrumentos facilitadores do processo online [Khan 2013].

A relação homem-máquina com a inserção da internet trouxe novos conceitos ao cotidiano da sociedade, por exemplo, mundo virtual, redes de relacionamento [Peña e 
Allegretti 2012] e acesso as informações organizadas em navegação não linear [Aquino 2007]. E com essa estratégia de aprendizagem híbrida se busca tornar os alunos autônomos, críticos e flexíveis que são capazes de interpretar, lidar com bibliografia e realidade, ainda alcançar pensamento crítico [Russo et al. 2013].

Entre as vantagens apontadas por [Kose 2010] na aplicação desta abordagem de ensino aprendizagem lista-se: acesso às informações de qualquer lugar, alunos de níveis e diferentes estilos de aprendizagem no mesmo espaço [Khan 2013] e o uso de várias técnicas de ensino atrair a atenção dos alunos. Há o aumento na interação entre os alunos e com o professor [Mohammad 2009]. Essa geração de alunos denominada nativo digital, nascida em meio à inserção de tecnologias de informação e comunicação no cotidiano, com essa característica dessa geração justifica-se o uso de sistemas e ferramentas digitais também no ambiente escolar [Khan 2013].

Esse ensino misto tem como aspecto de destaque ofertar conhecimento para o aluno no tempo apropriado com a tecnologia favorável, através de estilo de ensino adequado por meio de métodos [Yongxing 2008]. Entre as técnicas que podem ser utilizadas nesse método nos momentos presenciais cita-se: coaching, participação em um curso, participação em um seminário, workshops e, obviamente, aulas expositivas. Já nos momentos virtuais (online) podem usar como ferramentas as mídias sociais, aprendizagem móvel e gamificação. Neste trabalho destacasse as ferramentas online.

\subsection{Mídias Sociais}

As mídias sociais são os espaços colaborativos em que ocorrem compartilhamento de informações, opiniões e experiências, onde o usuário não é apenas observador, mas é o produtor de conteúdo. Entre os muitos exemplos de mídias sociais cita-se blog, microblog, fórum, wiki, podcasts, rede social e sites de compartilhamento de arquivos multimídia.

Evidencia-se a rede social como uma tendência para partilhar informações e conhecimento [Patrício e Gonçalves 2010], a qual é definida como conjunto de atores e suas conexões. Na qual se observa os padrões de conexão, a partir das diversas interações estabelecidas entre os atores sociais. O conceito de rede social surgiu antes do fenômeno da internet. Em 1736 foi publicado um artigo que abordava o enigma de Pontes de Königsberg, que deu origem ao primeiro teorema da teoria dos grafos - parte da matemática aplicada. Um grafo representa uma rede em diversos sistemas através de nós e arestas [Recuero 2009].

As redes sociais ganham espaço em vários setores da sociedade, transformam a maneira como as pessoas interagem, apresentam ideias e informações, e até como julgam a qualidade dos conteúdos e contribuições. Quase $40 \%$ da população mundial usam essas ferramentas no cotidiano [Johnson et al 2014].

Em um site de rede social como o facebook há diversas conexões de interações entre os atores, que representam o curtir, compartilhar, comentar, publicar, cutucar, aceitar ou recusar amigos e chat. A rede social é o que já acontecia offline, são as interações entre pessoas, que sem a tecnologia ficavam a conhecer um número mais reduzido de pessoas e culturas [ Garcia e Ferreira 2011]. 
O acesso computacional ampliou a possibilidade de interação, que pode ocorrer assíncrona (atores interagindo em momentos diferentes) ou síncrona (atores online interagindo ao mesmo tempo). E a interação pode ser mútua ou reativa linear, segundo proposta de estudo de [Primo 2000] que defende esses conceitos respectivamente como sistema aberto e sistema fechado. O sistema aberto é caracterizado por apresentar relação interdependente, onde um recebe influência e o sistema todo modifica, ou seja, o contexto influencia o sistema que tem trocas constantes entre todos. Enquanto que o sistema fechado tem relações lineares e unilaterais, com escolhas pré-determinadas, seguindo roteiro. Por exemplo, chats promovem interação mútua, enquanto que enquetes promovem interação linear.

As mídias sociais impactam mudanças no sistema escolar e provocam o repensar sobre o ensino tradicional. Essas tecnologias são acessíveis e tem custo baixo. Os sites de redes sociais são bastante representativos para o contexto educacional, visto que há familiaridade com uso dessa ferramenta. E o acesso a internet no Brasil está cada vez maior, como se pode confirmar por meio das pesquisas apresentadas pelo IBGE Instituto Brasileiro de Geografia e Estatística [IBGE 2013] que indicam o crescimento no acesso dos jovens a internet e tenta traçar o perfil do internauta:

Os estudos mostraram que a participação dos estudantes entre aqueles que
utilizaram a Internet reduziu de $43,4 \%$, em 2005 , para $35,1 \%$, em 2011 .
Explica-se que tal redução ocorreu, principalmente, em função de dois
fatores: maior aumento de usuários entre os não estudantes (aumento de
179,7\%) e queda de $3,2 \%$ observada no contingente total de estudantes, esta
devido ao envelhecimento da população [IBGE 2013].

As mídias sociais são novos modelos de ambientes de comunicação inseridos no cotidiano. E que formam possibilidades de estreitar o processo de ensino aprendizagem ao Ensino Híbrido que transpassa a sala de aula física e que pode atingir mais pessoas.

\subsection{Gamificação}

A gamificação consiste no lúdico através do uso de mecanismos de jogos com o objetivo de resolver problemas ou de despertar engajamento entre um público específico sobre um determinado assunto [Medina et al. 2013]. Há diversas experiências sobre o uso e criação de games e jogos no ambiente escolar. [McGonigal 2012] apresenta quatro características que definem um jogo:

- Meta: o objetivo do jogo;

- Regras: limitações para atingir o objetivo do jogo;

- Sistema de feedback: informações de quanto ainda falta para atingir a meta;

- Participação voluntária: aceitação consciente e voluntária da meta, regras e do feedback.

A Gamificação usa dessas características e do design de jogos inseridas em desafios para motivar pessoas a resolver problemas, participarem de treinamento, melhorar o aprendizado, uma maneira divertida e criativa [França e Reategui 2013]. 


\subsection{Aprendizagem móvel}

O cenário da aprendizagem móvel (M-Learning) consiste em uso de celulares, i-pods, rádio, TV ou tablet. É um modelo de ensino a distância por meio de tecnologias de redes móveis que atendem pessoas em mobilidade. O M-Learning é a união de tecnologias de processamento e comunicação de dados móveis e portáteis que permitem maior interação [Mozzaquatro et al. 2010]. As mídias sociais, softwares educacionais ou plataformas estão sendo adaptadas ou criadas para funcionarem nos aparelhos móveis e assim alavancar projetos de ensino aprendizagem.

\section{Curso Híbrido de Programação de Computadores}

O objetivo geral do curso foi de capacitar os alunos a visualizarem soluções computacionais para problemas através da construção de softwares na linguagem de programação C. Enquanto que os específicos foram de apresentar os conceitos básicos da linguagem de programação $\mathrm{C}$, contribuir na preparação do aluno no processo básico de desenvolvimento de software (concepção, edição, execução e teste de programas de computador) e para ele estar apto a participar da Olimpíada de Programação.

$\mathrm{O}$ curso de ensino de programação de computadores em linguagem $\mathrm{C}$ foi realizado inicialmente com 22 alunos de ensino médio da primeira a terceira série, sendo que dois alunos desistiram nas primeiras aulas, devido conflito de horário com outras atividades. O curso básico ocorreu no período de 23 de setembro a 5 novembro de 2013, enquanto que o curso avançado foi no período de 6 de novembro a 19 de dezembro de 2013.

Para realização do curso foi elaborada uma cartilha sobre programação de computadores, slides de apresentação do conteúdo do curso, vídeo aulas e foram desenvolvidas listas de exercícios, conforme a necessidade da turma.

O curso contemplou os seguintes assuntos:

a) Básico: História do computador; Introdução a Linguagem C; Variávéis e Expressões; Controle de Fluxo; Vetores, Strings e Matrizes.

b) Avançado: Ponteiros e Funções.

\subsection{Ambiente Presencial}

No primeiro momento presencial foi apresentada a área da ciência da computação, os cursos relacionados como engenharia da computação, ciência da computação, sistemas de informação entre outros. As aulas foram realizadas de segunda a sexta no laboratório de informática da escola de ensino médio, duas horas diárias, em período diferente do escolar. Através dos slides eram apresentados os assuntos e exemplos de exercícios, que em seguida eram realizados no laboratório juntamente com novos exemplos que eles precisavam iniciar e concluir.

\subsection{Ambiente Online}

A rede social "facebook" foi selecionada para atender a demanda de compartilhamentos das atividades, os materiais do curso, para esclarecer as dúvidas, os links para instalação de softwares necessários para que pudessem replicar ou aperfeiçoar as atividades em outros computadores fora do espaço de sala de aula. E também ser o meio de 
comunicação entre alunos e o professor (aluno de graduação de Engenharia da Computação), assim como com os demais colaboradores da universidade. Os vídeos foram disponibilizadas no "youtube", mas os links foram divulgados no grupo do facebook.

\subsection{Projeto Interdisciplinar}

Elaborou-se uma lista de projetos com 10 exercícios de programação que atendiam algumas das informações fornecidas pelos próprios alunos em um questionário sobre o perfil deles aplicado ainda no início do curso. Os exercícios envolviam diversos assuntos como biologia, geografia, trânsito, reciclagem entre outros, exemplificados na figura 1

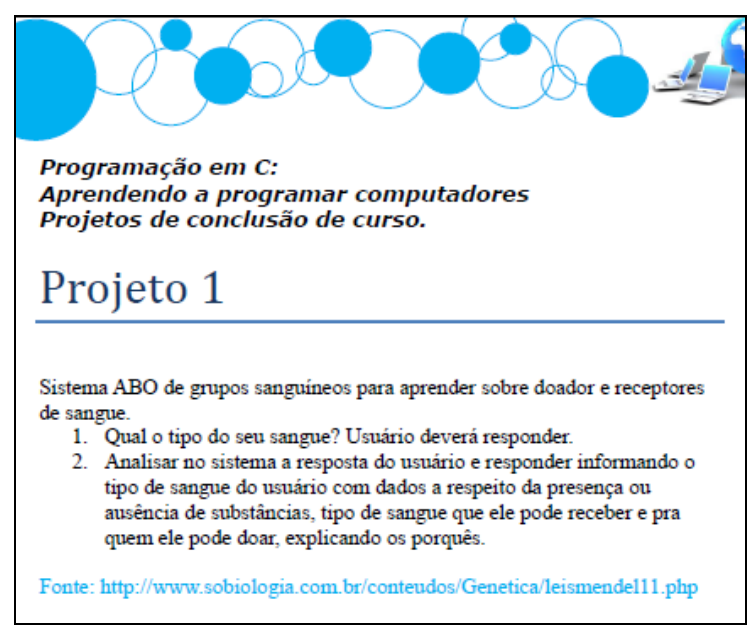

Figura 1. Exercício da lista dos projetos

Os alunos desenvolviam o projeto da lista de exercícios e compartilhavam no grupo do facebook, para o professor corrigir e os colegas também baixarem para testarem.

\section{Resultados e Contribuições}

Foram certificados 16 alunos do total de 22 inscritos no curso. Ocorreu a participação de três alunos do ensino fundamental. A faixa etária desses jovens está entre 16 a 25 anos. Esses alunos já tinham familiaridade com o uso do computador, porém não tinham muita informação sobre os cursos de graduação. Alguns já haviam lido por curiosidade sobre linguagem de programação, mas ainda não tinha realizado nenhuma atividade. Um dos aspectos positivo da aplicação do curso foi à aprovação de um jovem na graduação no curso de engenharia da computação em uma universidade local.

Ainda na busca em compreender essa pesquisa piloto, realizou-se um estudo mais avançado em função das interações realizadas no grupo da rede social facebook, que tem acesso fechado, restrito apenas aos participantes do curso presencial.

Em uma breve análise no grupo do facebook há 26 membros, sendo que 21 são os alunos iniciaram participação no curso e os demais são colaboradores da pesquisa e o aluno de graduação que foi o professor do curso. E foram compartilhados 45 arquivos entre material e respostas de exercícios. 
O software NodeXL associado ao plugin Social Network Importer foram utilizados para baixar os dados do grupo do facebook e depois realizar a análise da rede social. Encontrou 1889 interações no grupo que envolvia ações de curtir, comentar e visualização das postagens e do perfil dos usuários do grupo. E gerou um grafo da rede que apresenta 20 nós (vértices) que se ligam através 72 conexões (arestas) únicas como pode ser visualizada através da figura 2. A densidade dessa rede é de 190 conexões possíveis. Que significam que ocorreram até o momento desse levantamento de dados da pesquisa apenas $37,8 \%$ do total de conexões dessa rede que a classifica de baixa densidade. $\mathrm{O}$ tamanho dos nós está diretamente ligado ao grau de conexão na rede. $\mathrm{O}$ conjunto de conexões é representado pelos graus: $2,3,5,7,8,9,10,11,13,14,15,18$ e 19. Para identificar os atores dessa rede, os nós foram coloridos em vermelho representando os alunos ativos no grupo do facebook ( com os graus de conexão: 3, 5, 7, $8,9,10,11,13,14,18)$ e os nós azuis a equipe do projeto (com os graus:. 19, 15, 13, 2).

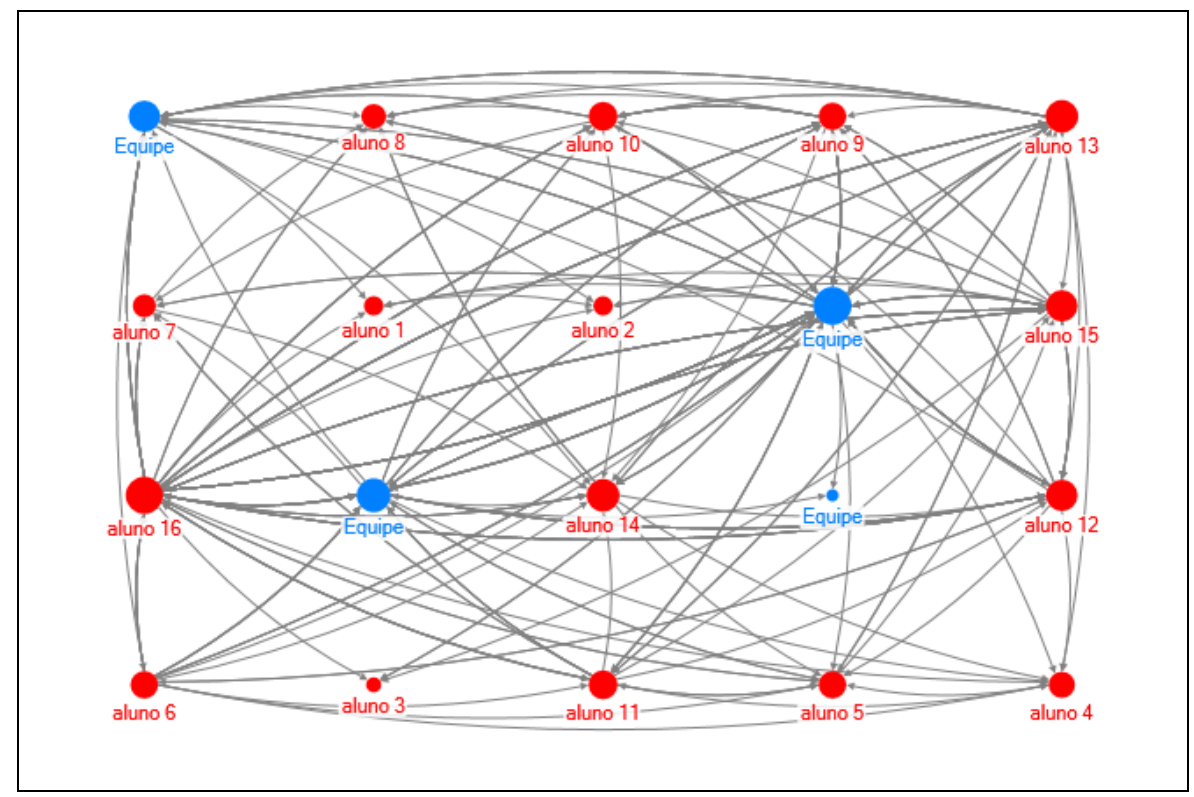

Figura 2. Grafo da Rede do Curso de Programação

Essas relações foram possíveis de serem encontradas a partir dos dados analisados. E ainda com base nesses dados obtiveram-se outras métricas de análise geral da rede.

a) A Centralidade de Proximidade (Closeness Centrality) é uma métrica do grau de proximidade entre os nós e mede a independência em relação ao controle dos outros [Marteleto 2001]. No experimento realizado o mínimo foi de 0,28 e o máximo de 0,53 de centralidade de proximidade entre os nós.

b) Grau de Entrada e Saída (in degree e out degree) são métricas do grau de centralidade de um nó que encontram alguns índices relacionados a influência direta de um vértice em relação aos seus contatos [Freitas 2010]. Que no experimento apresentou para o grau de entrada o mínimo 2 e o máximo 9 , enquanto para o grau de saída o mínimo 0 e o máximo 18.

c) Centralidade de Intermediação (Betweenness Centrality) que significa o potencial dos intermediários na rede, ou seja a importância na 
mediação[Marteleto 2001]. Apresentou os seguintes dados, o mínimo da centralidade de intermediação foi zero e o máximo foi $57,755$.

O aluno 16, conforme análise dos dados da rede apresentou entre os alunos a maior centralidade de proximidade com o índice de 0,050; centralidade de intermediação com índice de 36,755; grau de entrada igual a 5 e grau de saída igual a 18. Porém destacasse que o aluno 3 com maior frequência no curso presencial é o que menos interagiu na rede. Mas os alunos 1 e 2 que não concluíram o curso permaneceram no grupo, ainda que com pouca interação. Um aluno que não aparece no grafo está no grupo do facebook, mas não fez nenhuma interação com os demais participantes no curso online, porém participou nos momentos presenciais. Os alunos ficam livres para permanecerem ou não no grupo, porém o administrador aceita ou não inserir novos membros.

Com base nessas informações e nas solicitações que recebemos no grupo de pessoas interessadas em participar deve ser repensada a proposta de abrir o grupo fechado no facebook para atender uma maior interação na rede consequentemente maior troca de conhecimento. A interação pode ser mútua ou reativa linear na rede social, conforme as ferramentas utilizadas. $\mathrm{O}$ sistema aberto ainda é o mais atrativo por gerar uma relação interdependente, onde há trocas constantes de conhecimento.

\section{Considerações Finais}

O processo de análise de rede foi realizado de maneira mais geral, conforme o resultado processado no software NodeXL. A baixa densidade identificada representa que há possibilidade de crescimento nas conexões da rede. Sabe-se da necessidade de usar mecanismos de incentivo para participação em comunidades virtuais com base em manter viva a rede.

A tecnologia na educação precisa de um acompanhamento de uma nova cultura de ensino aprendizagem que vá além do ensino tradicional, buscando atender a nova realidade do cotidiano dos atores envolvidos nesse processo [Peña e Allegretti 2012]. Ter um espaço na rede permite ampliar o processo de aprendizagem que passa a ser continuo enquanto estiverem interagindo. Realizar o curso híbrido pode facilitar também interações com outros profissionais que irão acrescentar mais conhecimento e experiência na rede. E a análise servirá para descobrir os perfis dos alunos usando esse tipo de tecnologia e como o professor poderá intervir para que o curso seja motivador.

Atualmente o curso presencial foi encerrado. Mas a rede continua ativa com as publicações relacionadas a área da computação. O curso foi realizado com intuito de atrair novos alunos para a área da computação. Ocorreram 17 solicitações de usuários do facebook para participarem do curso online.

O curso motivou os alunos a buscarem mais informações a respeito da área da computação e a rede ficou como um canal de comunicação, onde entram em contato com a equipe para saber sobre novos cursos ou informações relacionadas a área da computação. Em cursos com período a longo prazo poderá ser mais fácil obter as métricas, intervir com ações motivacionais e acompanhar os resultados.

Existe a pretensão de ampliar os estudos das métricas geradas pela análise do software NodeXL para compreender ainda mais cada indivíduo participante do processo 
de aprendizagem em ensino híbrido. Confrontando os dados também da análise realizada em sala de aula. Buscando compreender novas possibilidades de ensino aprendizagem na área da computação como aspectos motivacionais.

\section{Referência}

Aquino, M. C. (2007). Hipertexto 2.0, folksonomia e memória coletiva: um estudo das tags na organização da web. Revista e-compós. v. 18. n. 18.

Bennedsen, J.; Caspersen, M. E. (2009). Recalling Programming Competence, Proceedings do 9th Koli Calling International Conference on Computing Education Research, Koli National Park, Finland. p. 86-95.

Falavigna, M. S. (2011). Inclusão digital: vivências brasileiras. São Paulo: IPSO Instituto de Projetos e Pesquisas Sociais e Tecnológicas. 250 p.

França, R. M.; Reategui, E. B. (2013). SMILE-BR: aplicação de conceitos de gamificação em um ambiente de aprendizagem baseado em questionamento. In: Anais do Simpósio Brasileiro de Informática na Educação, 24, Campinas, Brasil, p. 366-375.

Freitas, L. Q. (2010). Medidas de Centralidade em Grafos. Tese de Doutorado. Universidade Federal do Rio de Janeiro.

Garcia, L.; Ferreira, M. J. (2011). A rede social Facebook enquanto ferramenta de suporte ao ensino colaborativo/cooperativo. Revista do Departamento de Inovação, Ciência e Tecnologia, 2/3, p. 71-77.

IBGE - Instituto Brasileiro de Geografia e Estatística (2013). Pesquisa Nacional por Amostra de Domicilio: Acesso à Internet e Posse de Telefone Móvel Celular para Uso Pessoal 2011. Rio de Janeiro.

Johnson, L.; Adams Becker, S.; Cummins, M.; Estrada, V.; Meira, A. (2012). Technology Outlook for Brazilian Primary and Secondary Education 2012-2017: An NMC Horizon Project Sector Analysis. Austin, Texas: The New Media Consortium.

Johnson, L.; Adams Becker, S.; Estrada, V.; Freeman, A. (2014). NMC Horizon Report: 2014 Higher Education Edition. Austin, Texas: The New Media Consortium.

Kenski, V. M. (2008). Educação e Comunicação: Interconexões e Convergências. Educação \&. Sociedade, Campinas, vol. 29, n. 104 - Especial, p. 647-665.

Khan, Z. R. (2013). Blogging and online collaborative discovery learning: making a case for a successful group-tracking technique. In Int'l Conf. Frontiers in Education: $\mathrm{CS}$ and $\mathrm{CE}$.

Kose, U. (2010). A blended learning model supported with Web 2.0 technologies. In Procedia Social and Behavioral Sciences 2, p. 2794-2802.

Lima, A. P. L. (2014). O uso de ferramentas da Web 2.0 no compartilhamento de Informação e Conhecimento. Revista do Mestrado Profissional Gestão em Organizações Aprendentes, v. 3, n. 1, p. 128-139.

Lobo, M. B. de C. M. (2012). Panorama da evasão no ensino superior brasileiro: aspectos gerais das causas e soluções. ABMES Cadernos. Brasília. 
Maness, Jack. (2006) Library 2.0 Theory: Web 2.0 and its implications for libraries. Webology, v.3, n.2, Artigo 25. Disponível em: http://www.webology.ir/2006/v3n2/a25.html. Acesso em: 11 de jul. 2014.

Marteleto, R. M. (2001). Análise de redes sociais: aplicação nos estudos de transferência da informação. Ciência da informação, v. 30, n. 1, p. 71-81.

McGonigal, Jane (2012). A realidade em jogo. Tradução de Eduardo Rieche. Rio de Janeiro: BestSeller. 378 p.

Medina, B.; Tanaka, S.; Vianna, M.; Vianna, Y. (2013). Gamification, Inc.: como reinventar empresas a partir de jogos. 1. ed. Rio de Janeiro: MJV Press. 118 p.

Mohammad, F. (2009). Blended Learning and the Virtual Learning Environment of Nottingham Trent University. In Second International Conference on Developments in eSystems Engineering. p. 295- 299.

Mozzaquatro, P. M.; Franciscato, F. T.; Ribeiro, P. S.; Medina, R. (2010). Ambiente Virtual de Aprendizagem Móvel adaptado aos diferentes estilos cognitivos utilizando Hipermídia Adaptativa. In Anais do Workshop sobre Informática na Escola. 16. Belo Horizonte. Brasil. p. 1255-1264.

Nascimento, A.D., and Hetkowski, T.M.,.(2009) Educação e Contemporaneidade: pesquisas científicas e tecnológicas [online]. Salvador: EDUFBA, $401 \mathrm{p}$.

Patrício, M. R.; Gonçalves, V. (2010). Facebook: rede social educativa?. In: Encontro Internacional TIC e Educação. 1. Lisboa, Portugal, p. 593-598.

Peña, M. D. J.; Allegretti, S. M. M. (2012). Escola Híbrida: aprendizes imersivos. Revista Contemporaneidade, Educação e Tecnologia. São Paulo, v. 1, n. 2, p. 97-107, abril/2012. Disponível em: http://revistacontemporaneidadeeducacaoetecnologia02.wordpress.com/. Acesso: 11 jul. 2014.

Primo, A. F. T. (2000). Interação mutua e interação reativa: uma proposta de estudo. Revista FAMECOS: mídia, cultura e tecnologia. v. 1, n. 12, p. 81-92.

Recuero, R. (2009). Redes Sociais na Internet. Porto Alegre: Sulina. 191 p.

Russo, C.; Sarobe, M; Ramón, H.; Alonso, N.; Esnaola, L.; Lencina, P.; Ahmad, T. (2013). Methodology strategies for a University entrance course: Coaching and tutoring, the experience at the National University of the North West of the Province of Buenos Aires, Argentina. In Int'l Conf. Frontiers in Education: CS and CE.

Scaico, P. D.; Queiroz, R. J. G. B. (2013). A educação do futuro: uma reflexão sobre aprendizagem na era digital. In: Simpósio Brasileiro de Informática na Educação. 24. Campinas. Brasil. p.

Yongxing, W. (2008). Blended Learning Design for Software Engineering Course Design. International Conference on Computer Science and Software Engineering. p. 345-348. 